\title{
Experimental and numerical study of the damage mechanisms in hybrid unidirectional/woven composites under impact loading
}

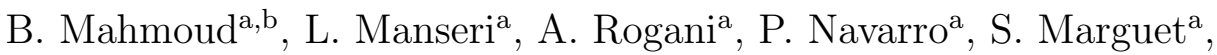 \\ J-F. Ferrero ${ }^{\mathrm{a}}$, I. Tawk ${ }^{\mathrm{b}}$ \\ ${ }^{a}$ Université de Toulouse, Institut Clément Ader, FRE CNRS 3687, \\ UPS/INSA/ISAE/Mines Albi, 3 rue Caroline Aigle, 31400 Toulouse, France \\ ${ }^{b}$ University of Balamand, Deir El-Balamand, El-Koura, Lebanon
}

\begin{abstract}
This paper deals with low velocity and medium velocity impacts on thin carbon/epoxy hybrid laminates made of unidirectional plies and woven fabric layers. Drop weight and gas gun impact tests were performed to compare the damage mechanisms of thin hybrid laminates with those of woven laminates of equivalent stiffness and mass. The results show that hybrid laminates present a better impact behaviour in terms of post impact damage extent. These tests were then modeled using a semi-continuous approach. The numerical results well correlate the experiments.
\end{abstract}

Keywords: Hybrid composite, Damage, Impact, Explicit F.E modeling

\section{Introduction}

This article deals with the impact behaviour of thin carbon/epoxy hybrid composite laminates made of unidirectional and fabric layers. The work focuses on experimental analysis of damage mechanisms and on the development of a specific finite element model able to predict damage mechanisms 
and chronology during low and medium velocity impacts.

Composite materials are widely used in many applications, especially where high strength and stiffness to weight ratio is concerned. This material characteristic is interesting for a wide range of industries, particularly in the transport industry such as aircrafts, helicopters, boats or cars.

However, when impacted, composite laminates exhibit a relatively brittle behavior with extensive matrix cracking, delamination or fibers breakages [1]. This damage can lead to a loss of stiffness and eventually a loss of load carrying capability when fibers break. For this reason, impact behavior of unidirectional or woven composite laminates has been widely studied experimentally to understand the impact phenomena and the role of different parameters on impact damage. These investigations, performed for different materials and configurations, have been well summarized in different review papers $[1,2,3]$. The most influencing parameters are the specimen stacking sequence $[4,5]$, the specimen geometry $[6,7]$, the nature of fibres and matrix $[8,9,10]$, the impactor mass, size and geometry $[11,12]$ and the impact energy [13]. The architecture of the reinforcement is also a key parameter $[14,15]$. Indeed, for woven laminates, the measured peak loads and damage areas are lower than for unidirectional laminates.

Concerning the modelling of impacts on composite laminates, several approaches have been formulated [16]. They can be divided in differents parts. The fracture mechanics approaches $[17,18]$ provide interesting results but causes difficulties like a sensitivity to the mesh size. The damage mechanics approaches [19, 20, 21, 22] successfully predict matrix cracking and delamination responsible for stiffness degradation. Delamination is usually made 
using interface elements [23, 24]. Wisnom [25] and Abrate [26] have provided detailed reviews on the use of cohesive zone interface elements to model matrix failures in polymer composites. Another modelling strategy is the Semi-Continuous approach $[27,28,29,30,31,32]$. Damage mechanisms in woven and unidirectional laminates under low and medium velocity impacts are well predicted.

In the field of hybrid laminates, many papers were published. Most concerns laminates that combine two or more different existing materials [33]. In the comprehensive review provided by Swolf [34], fibre hybridization studies are presented. Generally, three main scales are used for the hybridization : the layer scale, the bundle scale and the fiber scale. Many positive hybrid effects were found, especially when dealing with simple loading like tensile loading. As far as more complex loading is concerned, for example impacts, Swolf concludes that mechanisms are less understood, with sometimes contradictions for the conclusions of different studies [35, 36, 37]. However, recent work [38] shows that using hybridization to maximize the translaminar fracture toughness can increase the penetration impact resistance of laminates. Concerning the specific topic of impacts on panels hybridized with the same fiber and matrix material, but with different architectures for the layers (for example by mixing unidirectional and fabric layers), few papers were found. The studies found deal with ballistic performance of thick hybrid composite panels for body armors $[39,40]$ or low velocity impact on UD composite laminate positioned between two ply of 5HS fabric carbon to protect it [41].

In this paper, an experimental study is carried out to show the potential of thin hybrid unidirectional/woven laminates compared to woven laminates. 
Gas gun and drop weight tests are performed on hybrid and woven thin laminates of equivalent stiffness. The respective damages are analyzed and compared. The tests are modeled with the Semi-Continuous strategy. The numerical results well correlate the experiments.

\section{Experimental study}

In this part, prospective tests on hybrid UD/woven composite laminates are presented. Low velocity and medium velocity impact responses of hybrid samples and woven laminates are compared. Four configurations, presented in Figure 1, are studied.

The two woven laminate samples are made with three plies of carbon/epoxy woven fabric. Two plies are oriented at $\pm 45^{\circ}$ and one ply at $0^{\circ} / 90^{\circ}$. For the first configuration (W1), the $0^{\circ} / 90^{\circ}$ ply is at the bottom. For the second one (W2), the $0^{\circ} / 90^{\circ}$ ply is at the middle. The hybrid samples (H1 \& H2) have almost the same stacking sequence than the woven samples, except that the $0^{\circ} / 90^{\circ}$ ply is replaced by three unidirectionnal layers oriented at $\left[0^{\circ} / 90^{\circ} / 0^{\circ}\right]$. The thickness is the same for the four samples : $0.95 \pm 0.01 \mathrm{~mm}$. The hybrid configurations were chosen to have the same stiffness than the woven laminates.

\subsection{Low velocity impacts}

Drop weight impact tests were conducted. Three specimens per configuration were impacted. The sample dimensions are $100 \times 125 \mathrm{~mm}$. They are positioned on a rigid frame with a dimension of $75 \times 100 \mathrm{~mm}$, as described in Figure 2. The steel impactor has a $16 \mathrm{~mm}$ diameter hemispherical shape and a mass of $2 \mathrm{~kg}$. It impacts the specimen with an initial velocity of $2 \mathrm{~m} / \mathrm{s}$, that 
is to say an impact energy of $4 J$. The reaction force and the displacement of the impactor are recorded during impact. Figure 3 and Figure 4 present respectively the load / displacement curves for the H1 and W1 samples and for the $\mathrm{H} 2$ and $\mathrm{W} 2$ samples.

The stiffness is the same for the four laminates. For the samples H1 and $\mathrm{W} 1$, for which the $0^{\circ} / 90^{\circ}$ plies are at the bottom, a difference in the rupture scenario is observed. Indeed the hybrid laminate presents a clear drop of load while the woven specimen failure is in two steps: a drop followed by a plateau. Moreover, the peak load is $27 \%$ higher for the hybrid sample. The absorbed energies, that corresponds to the area under the curves, are almost the same: the difference is $3.6 \%$.

When the $0^{\circ} / 90^{\circ}$ plies are at the middle, which corresponds to samples $\mathrm{H} 2$ and W2, the failure behaviour is the same. A drop of the load is first observed. It is followed by a plateau. The peak load is $28 \%$ higher for the hybrid sample. The absorbed energy is $37 \%$ higher for the woven laminates.

Figure 5 presents the post impact damages of the four samples. Fore the sake of visibility, the cracks are underlined with yellow dotted lines. On the impacted side, the damage size is the same except for configuration $\mathrm{H} 2$. Indeed, for the three other configurations, a residual dent and small cracks and fibers failure are observed while $\mathrm{H} 2$ presents a barely visible residual dent and no visible crack or failure. The damage extent observed on the opposite side from the impact varies with the configuration. The two woven samples present fractures with approximately the same size. Sample H1 presents the biggest damage : a cross with a size $110 \%$ higher than the ones observed in W1 and W2 bottom plies. The damage in the lower surface of $\mathrm{H} 2$ is the 
smallest, it is $15 \%$ lower than what measured on samples W1 and W2.

A correlation can be found between the size of the fracture surface and the absorbed energy measured in Figure 3 and Figure 4. Indeed, sample H2 that presents less fiber failure is the sample that has absorbed the less energy.

Furthermore, these results show that the substitution of a woven ply by unidirectional plies in a thin woven laminate has beneficial effects on the low velocity impact response. It is all the more interesting when the unidirectional plies are at the middle.

This behaviour can be explained by the optimal combination of the advantages provided by the architecture of the constitutive layers, which compensates their drawbacks. Typically, unidirectional plies present very good mechanical properties in the direction of the fibers, but are weaker in the transverse direction. The consequence is the apparition of large intralaminar transverse cracks and delamination when impacted. On the contrary, for woven composite fabrics, propagation of large intralaminar cracks is prevented by the weaving of the bundles of fibers. However, this particular architecture results in poorer mechanical properties than unidirectional layers. Hence protecting the unidirectional plies between two plies of fabric layers make it possible to have a thin laminate that combines good mechanical properties and an improved resistance to impact loading.

\subsection{Medium velocity oblique impacts}

Medium velocity oblique impact tests were performed with a gas gun device. The specimens are square with a size of $200 \times 200 \mathrm{~mm}$. They are simply supported on two edges, as described on Figure 6 . The impactor 
is a $19 \mathrm{~mm}$ steel ball with a mass of $28 \mathrm{~g}$. The impact angle is $15^{\circ}$. The velocity of the impactor is $80 \mathrm{~m} / \mathrm{s} \pm 4 \mathrm{~m} / \mathrm{s}$. Three tests were performed for each configuration.

Figure 7 and Figure 8 show the fracture surfaces observed on the top and bottom plies after impact. The oblique impact damage shape presents some particularities, as described in [42]. It is different to the fracture surface resulting from a low velocity normal impact which has a cross shape. Indeed, it is extended in the firing axis direction. When the ply is oriented at $0^{\circ} / 90^{\circ}$, the damage is a longitudinal crack. When it is oriented at $\pm 45^{\circ}$, the cracks initiate with a "V" shape and propagates with two parallel cracks oriented in the firing direction.

For the configurations $\mathrm{W} 1$ and H1, no damages are visible in the top ply. In the bottom ply of W1, a large crack is observed while in the bottom ply of the hybrid laminate H1, only a few resin cracks parallel to the fiber are observed.

For the configurations $\mathrm{W} 2$ and $\mathrm{H} 2$, the fracture surface observed in the bottom ply has the same shape and size. Nevertheless, the upper side of the woven sample presents a longitudinal crack while in the top ply of the hybrid sample, no damage is visible.

These visual observations confirm what was observed for low velocity impacts: the impact behaviour of hybrid laminates is better than the impact response of woven laminates with equivalent stiffness and mass. 


\section{Semi-Continuous modelling}

In this part, the modelling strategy used to model the impacts presented in the previous part is described. The detailed formulation is given in $[27,28$, 29, 30, 31, 32]. It has been developed in the explicit Finite Element software Radioss.

The main idea of the Semi-Continuous strategy is to represent the damage mechanisms observed experimentally by the use of a mesh size at the scale of the structure of the composite.

\subsection{Woven fabric modelling}

The principle is to model the woven fabric bundles with a truss structure which follows the woven pattern geometry. The mesh size respects the woven fabric pattern i.e. the distance between two bundles. The interlacing of the warp and the weft tows at the crimp regions is approximated with oblique rod elements. The epoxy matrix which stabilizes the fibre bundles is modeled with damageable shell elements placed at the neutral axis of the ply. The nodes of the rods are offset from the shell to a distance of $\frac{h}{4}$ in the thickness-direction where $\mathrm{h}$ is the ply thickness. Finally, the connection between the rods and the shell elements is realized at the nodes through rigid

links as exposed at the bottom-right side of Figure 9. Thus, the rotational degrees of freedom of the shell induce the tension and compression of the rods. This strategy allows to represent both the continuous elastic behavior of the undamaged ply and the discrete behavior of the woven ply when resin is damaged and fibre bundles are in a non-stabilized state. 


\subsection{Unidirectional ply modelling}

The modelling strategy for unidirectional plies relies on experimental observations of the failure under impacts. A fracture surface of an unidirectional thin laminate after impact is given in Figure 10.

The damage scenario can be described in three main steps. First, the resin is damaged. Longitudinal cracks propagating between the fibers are observed. The groups of fibers between these cracks, that constitute bundles of fibers, are not stabilized anymore by the resin. They behave independently, carrying mainly tensile and compressive loads. Finally, these bundles of fibers fail in tension when the ultimate strain of the material is reached. The modelling has to represent this damage scenario, and particularly unstabilized bundles. Thus, the proposed modelling strategy relies on a representation of the bundles of fibers with $1 \mathrm{D}$ rod elements, stabilized with a specific 2D shell element that can be damaged. The modelling scale is that of the size of the bundles of fibers: the mesh size corresponds to the distance between two longitudinal cracks. A description of the modelling is given in Figure 11.

\subsection{Interface modelling}

The laminate is built by connecting each unidirectional ply with specific shell-to-shell interface elements. The principle is presented in Figure 12.

These elements are 8-node elements with three translational and three rotational degrees of freedom per node. This allows feasible connection to shell elements. The idea is to take into account the thickness of the connected shells. Eight virtual nodes representing the physical interface are created. This interface is handled with a classic bilinear cohesive law that accounts for mode mixicity. The assumption is made that the straight lines normal 
to the midsurface of the plies remain normal, so that the virtual nodes are connected to the real nodes with rigid body elements. The reaction forces and momentum applied on the real nodes are deduced from the reaction forces applied on the virtual nodes.

\subsection{Identification}

The modelling is handled by numerous parameters for the elastic, pseudoplastic and damage behaviour. There are 12 parameters for the woven fabric modelling, 16 parameters for the unidirectional ply modelling and 5 parameters for the interface modelling. All these parameters are identified with a reverse method on several tests : tensile tests, compressive tests, three point bending tests, short beam tests, indentation tests, DCB and ENF tests. These tests are performed with static and dynamic loading. These experiments are then modeled, and the parameters are found in order to fit the experimental results (curves and damage extent). An example of reverse identification is given in Figure 13 for a DCB test.

\section{Results and discussion}

In this part, the tests presented in section 2 on the hybrid samples are modeled with the Semi-Continuous strategy on the explicit software Radioss. For the two types of impact, the impactor and the boundary conditions are represented by rigid surfaces. For the low velocity impact simulation, 88000 elements are used. The calculation time is 50 minutes on 160 cores. The oblique impact simulation is built with 280000 elements. The calculation time is 20 minutes on 160 cores. For both modelling, the mesh size is $1 \mathrm{~mm}$. 


\subsection{Low velocity impacts}

Figure 14 and Figure 15 show the load / displacement curves measured experimentally and given by the modelling for the samples $\mathrm{H} 1$ and $\mathrm{H} 2$.

A good correlation is found in terms of peak load and absorbed energy. The shape and size of the damage in the lower ply, where it is the highest, is given in Figure 16. The modelling well represents the damage scenario, the cross shape and the failure extent.

More, the numerical results are used to provide a better analysis of the damages within the laminates. Figure 17 and Figure 18 show the stress repartition in the unidirectional plies before the first damage and the final damage extent given by the modelling, respectively for samples H1 and H2. When the unidirectional plies are at the bottom (sample H1), they are mainly loaded in tension, which results from the global bending of the laminate. This induces the apparition of a large crack. When that crack opens, the fibers are loaded and break in tension. As a consequence, the final fracture shape is a large cross. When the unidirectional plies are at the middle (sample H2), they are loaded mainly in out of plane shearing. This shearing leads to the damage of the resin in a localized area. As the fibers are at the middle of the laminate and protected by the woven fabrics, the strain is lower than in the $\mathrm{H} 1$ case, so that very few fiber breakages are observed.

\subsection{Medium velocity oblique impacts}

The normal load versus time curves calculated and measured experimentally using Digital Image Correlation [43] are given in Figure 19 for H1 and in Figure 20 for H2. A peak load, followed by a plateau is observed. Numerical results well correlates the experimental curves. 
Figure 21 shows the numerical and experimental post impact damage of the lower ply of the hybrid samples.

A good correlation is found between experimental and numerical results. The biggest discrepancy is observed for the damage in the lower ply of H1. Nevertheless, the global shape of the damage is well represented, with the presence of multiple cracks perpendicular to the fire axis.

To understand the mechanisms leading to that particular fracture shape, the stress repartition in the plies, given by the model, is analyzed. Figure 22 gives the stress and damage state in the last ply during impact when the normal load is maximal, that is to say at the time corrsponding to the peak load. During impact, the last ply is mainly loaded in tension in the transverse direction (Figure 22 a). If the stress level is high enough, the matrix is damaged. Indeed, small cracks parallel to the fibers appear (Figure $22 \mathrm{~b}$ ). Nevertheless, even at the peak load, the stress in the fibers is not sufficient to provoke fiber breakages (Figure $22 \mathrm{c}$ ).

\section{Conclusion}

This article presents an experimental and numerical investigation on the behaviour of thin carbon/epoxy hybrid unidirectional/woven composite laminates under low and medium velocity impact loading. Four sample configurations are tested with a drop weight device and a gas gun in order to observe the behaviour of thin hybrid laminates and compare their response with a pure woven laminate with an equivalent stiffness. The main contribution of this experimental study is to show that an alternative to mixing different materials for the improvement of impact response of thin composite laminates 
is the hybridization using the same materials but different architectures. Indeed, the analysis of the impact tests results carried out in this paper reveals that it is possible to increase the impact resistance of thin carbon/epoxy laminates by putting unidirectional plies between woven fabric layers. The unidirectional plies are protected: the woven layers prevent the apparition of large intralaminar cracks.

The second main contribution of this paper is to validate the semi-continuous strategy for the modelling of impacts on thin hybrid samples. The modelling scale at the bundle pattern level and the decoupling between the fibers and the matrix makes it possible to represent the damage mechanisms and the local phenomena in the unidirectional plies and in the woven fabric layers, and that handle the impact response of the whole hybrid laminate. Hence, the analysis of these results are used to understand better the mechanisms leading to the final fracture shape.

\section{Acknowledgement}

The authors acknowledge the supercomputing centre CALMIP for granting access to the HPC resources under the allocation 2016-P09105.

\section{References}

[1] S. Abrate, Impact on composite structures, Cambridge University Press, 1998.

[2] C. Atas, O. Sayman, An overall view on impact response of woven fabric composite plates, Composite Structures 82 (3) (2008) 336-345. 
doi:10.1016/j.compstruct.2007.01.014.

URL http://www.sciencedirect.com/science/article/pii/S0263822307000177

[3] M. O. W. Richardson, M. J. Wisheart, Review of low-velocity impact properties of composite materials, Composites Part A: Applied Science and Manufacturing 27 (12) (1996) 1123-1131. doi:10.1016/1359835X(96)00074-7.

URL http://www.sciencedirect.com/science/article/pii/1359835X96000747

[4] S. A. Hitchen, R. M. J. Kemp, The effect of stacking sequence on impact damage in a carbon fibre/epoxy composite, Composites 26 (3) (1995) 207-214. doi:10.1016/0010-4361(95)91384-H.

URL http://www.sciencedirect.com/science/article/pii/001043619591384H

[5] E. Fuoss, P. V. Straznicky, C. Poon, Effects of stacking sequence on the impact resistance in composite laminates Part 1: parametric study, Composite Structures 41 (1) (1998) 67-77. doi:10.1016/S02638223(98)00036-1.

URL http://www. sciencedirect.com/science/article/pii/S0263822398000361

[6] G. Belingardi, R. Vadori, Influence of the laminate thickness in low velocity impact behavior of composite material plate, Composite Structures 61 (1) (2003) 27-38. doi:10.1016/S0263-8223(03)00027-8.

URL http://www.sciencedirect.com/science/article/pii/S0263822303000278

[7] P. Viot, L. Ballre, L. Guillaumat, J.-L. Lataillade, Scale effects on the response of composite structures under impact loading, Engineering Fracture Mechanics 75 (9) (2008) 2725-2736. 
doi:10.1016/j.engfracmech.2007.03.001.

URL http://www. sciencedirect.com/science/article/pii/S0013794407001166

[8] B. Vieille, V. M. Casado, C. Bouvet, Influence of matrix toughness and ductility on the compression-after-impact behavior of woven-ply thermoplastic- and thermosetting-composites: A comparative study, Composite Structures 110 (2014) 207-218. doi:10.1016/j.compstruct.2013.12.008.

URL http://www.sciencedirect.com/science/article/pii/S0263822313006296

[9] S. M. Bishop, The mechanical performance and impact behaviour of carbon-fibre reinforced PEEK, Composite Structures 3 (3) (1985) 295-318. doi:10.1016/0263-8223(85)90059-5.

URL http: //www.sciencedirect.com/science/article/pii/0263822385900595

[10] G. Dorey, S. M. Bishop, P. T. Curtis, On the impact performance of carbon fibre laminates with epoxy and PEEK matrices, Composites Science and Technology 23 (3) (1985) 221-237. doi:10.1016/02663538(85)90019-3.

URL http://www.sciencedirect.com/science/article/pii/0266353885900193

[11] T. Mitrevski, I. H. Marshall, R. Thomson, The influence of impactor shape on the damage to composite laminates, Composite Structures 76 (1) (2006) 116-122. doi:10.1016/j.compstruct.2006.06.017.

URL http://www. sciencedirect.com/science/article/pii/S0263822306002571

[12] W. J. Cantwell, J. Morton, The influence of varying projectile mass on the impact response of CFRP, Composite Structures 13 (2) (1989) 
101-114. doi:10.1016/0263-8223(89)90048-2.

URL http://www.sciencedirect.com/science/article/pii/0263822389900482

[13] W. J. Cantwell, J. Morton, Comparison of the low and high velocity impact response of cfrp, Composites 20 (6) (1989) 545-551. doi:10.1016/0010-4361(89)90913-0.

URL http://www.sciencedirect. com/science/article/pii/0010436189909130

[14] O. De Almeida, J.-F. Ferrero, L. Escal, G. Bernhart, Charpy test investigation of the influence of fabric weave and fibre nature on impact properties of PEEK-reinforced composites, Journal of Thermoplastic Composite Materials (2018) 0892705718778744doi:10.1177/0892705718778744. URL https://doi.org/10.1177/0892705718778744

[15] C. Evci, M. Glge, An experimental investigation on the impact response of composite materials, International Journal of Impact Engineering 43 (2012) 40-51. doi:10.1016/j.ijimpeng.2011.11.009.

URL http://www.sciencedirect.com/science/article/pii/S0734743X11001886

[16] G. Davies, R. Olsson, Impact on composite structures, Aeronautical Journal 108 (1089) (2004) 541-563.

[17] J. Whitney, R. Nuismer, Stress Fracture Criteria for Laminated Composites Containing Stress Concentrations, Journal of Composite Materials 8 (3) (1974) 253-265. doi:10.1177/002199837400800303. URL http://jcm.sagepub.com/cgi/doi/10.1177/002199837400800303

[18] E. Altus, A. Rotem, A 3-D fracture mechanics approach to the strength of composite materials, Engineering Fracture Mechanics 14 (3) (1981) 
637-649. doi:10.1016/0013-7944(81)90050-3.

URL http://linkinghub.elsevier.com/retrieve/pii/0013794481900503

[19] P. Ladeveze, G. Lubineau, An enhanced mesomodel for laminates based on micromechanics, Composites Science and Technology 62 (4) (2002) 533-541. doi:10.1016/S0266-3538(01)00145-2.

URL http://linkinghub.elsevier.com/retrieve/pii/S0266353801001452

[20] O. Allix, L. Blanchard, Mesomodeling of delamination: towards industrial applications, Composites Science and Technology 66 (6) (2006) 731-744. doi:10.1016/j.compscitech.2004.12.023.

URL http://linkinghub.elsevier.com/retrieve/pii/S026635380400363X

[21] L. Iannucci, J. Ankersen, An energy based damage model for thin laminated composites, Composites Science and Technology 66 (78) (2006) 934-951. doi:10.1016/j.compscitech.2005.07.033.

URL http://www.sciencedirect. com/science/article/pii/S0266353805003088

[22] F. Caputo, A. De Luca, G. Lamanna, V. Lopresto, A. Riccio, Numerical investigation of onset and evolution of LVI damages in CarbonEpoxy plates, Composites Part B: Engineering 68 (2015) 385-391. doi:10.1016/j.compositesb.2014.09.009.

URL http://www. sciencedirect.com/science/article/pii/S1359836814004119

[23] R. Borg, L. Nilsson, K. Simonsson, Simulation of low velocity impact on fiber laminates using a cohesive zone based delamination model, Composites Science and Technology 64 (2) (2004) 279-288. 
doi:10.1016/S0266-3538(03)00256-2.

URL http://linkinghub.elsevier.com/retrieve/pii/S0266353803002562

[24] P. P. Camanho, C. G. Davila, M. F. De Moura, Numerical Simulation of Mixed-Mode Progressive Delamination in Composite Materials, Journal of Composite Materials 37 (16) (2003) 1415-1438. doi:10.1177/0021998303034505.

URL http://jcm. sagepub.com/cgi/doi/10.1177/0021998303034505

[25] M. Wisnom, Modelling discrete failures in composites with interface elements, Composites Part A: Applied Science and Manufacturing 41 (7) (2010) 795-805. doi:10.1016/j.compositesa.2010.02.011.

URL http://linkinghub.elsevier.com/retrieve/pii/S1359835X10000680

[26] S. Abrate, J. F. Ferrero, P. Navarro, Cohesive zone models and impact damage predictions for composite structures, Meccanica 50 (10) (2015) $2587-2620$.

[27] P. Navarro, J. Aubry, S. Marguet, J. F. Ferrero, S. Lemaire, P. Rauch, Semi-continuous approach for the modeling of thin woven composite panels applied to oblique impacts on helicopter blades, Composites Part A: Applied Science and Manufacturing 43 (6) (2012) 871-879. doi:10.1016/j.compositesa.2012.01.020.

[28] P. Navarro, F. Pascal, J. Aubry, S. Marguet, J. F. Ferrero, S. Lemaire, P. Rauch, Semi-continuous approach for the study of impacts on woven composite laminates: Modeling interlaminar behavior with a specific 
interface element, International Journal of Impact Engineering 75 (2015) 184-193. doi:10.1016/j.ijimpeng.2014.08.012.

[29] F. Pascal, P. Navarro, S. Marguet, J.-F. Ferrero, On the modelling of low to medium velocity impact onto woven composite materials with a $2 \mathrm{~d}$ semi-continuous approach, Composite Structures 134 (2015) 302-310. doi:10.1016/j.compstruct.2015.08.067.

[30] B. Mahmoud, M. Colungo Torrecilla, P. Navarro, S. Marguet, I. Tawk, J.-F. Ferrero, Semi-continuous strategy for the modelling of damage mechanisms in unidirectional composites under low velocity impacts, Composites Part B: Engineering 130 (2017) 147-157. doi:10.1016/j.compositesb.2017.07.014.

URL http://www.sciencedirect.com/science/article/pii/S135983681730135X

[31] F. Pascal, A. Rogani, B. Mahmoud, P. Navarro, S. Marguet, J.F. Ferrero, Impact damage prediction in thin woven composite laminates Part II: Application to normal and oblique impacts on sandwich structure, Composite Structures 190 (2018) 43-51. doi:10.1016/j.compstruct.2018.02.013.

URL http://www.sciencedirect.com/science/article/pii/S0263822317343027

[32] F. Pascal, O. Dorival, P. Navarro, S. Marguet, J. F. Ferrero, Impact damage prediction in thin woven composite laminates Part I: Modeling strategy and validation, Composite Structures 190 (2018) 32-42. doi:10.1016/j.compstruct.2018.02.007.

URL http://www.sciencedirect.com/science/article/pii/S0263822317343015 
[33] M. R. Wisnom, G. Czl, Y. Swolfs, M. Jalalvand, L. Gorbatikh, I. Verpoest, Hybrid effects in thin ply carbon/glass unidirectional laminates: Accurate experimental determination and prediction, Composites Part A: Applied Science and Manufacturing 88 (2016) 131-139. doi:10.1016/j.compositesa.2016.04.014.

URL http://www.sciencedirect.com/science/article/pii/S1359835X16300896

[34] Y. Swolfs, L. Gorbatikh, I. Verpoest, Fibre hybridisation in polymer composites: A review, Composites Part A: Applied Science and Manufacturing 67 (2014) 181-200. doi:10.1016/j.compositesa.2014.08.027.

URL http://www.sciencedirect.com/science/article/pii/S1359835X14002681

[35] N. K. Naik, R. Ramasimha, H. Arya, S. V. Prabhu, N. ShamaRao, Impact response and damage tolerance characteristics of glasscarbon/epoxy hybrid composite plates, Composites Part B: Engineering 32 (7) (2001) 565-574. doi:10.1016/S1359-8368(01)00036-1.

URL http://www.sciencedirect.com/science/article/pii/S1359836801000361

[36] E. Sevkat, B. Liaw, F. Delale, B. B. Raju, Drop-weight impact of plainwoven hybrid glassgraphite/toughened epoxy composites, Composites Part A: Applied Science and Manufacturing 40 (8) (2009) 1090-1110. doi:10.1016/j.compositesa.2009.04.028.

URL http://www.sciencedirect.com/science/article/pii/S1359835X09001274

[37] E. V. Gonzlez, P. Maim, J. R. Sainz de Aja, P. Cruz, P. P. Camanho, Effects of interply hybridization on the damage resistance and tolerance of composite laminates, Composite Structures 108 (2014) 319-331. 
doi:10.1016/j.compstruct.2013.09.037.

URL http://www.sciencedirect.com/science/article/pii/S0263822313004844

[38] Y. Swolfs, Y. Geboes, L. Gorbatikh, S. T. Pinho, The importance of translaminar fracture toughness for the penetration impact behaviour of woven carbon/glass hybrid composites, Composites Part A: Applied Science and Manufacturing 103 (2017) 1-8. doi:10.1016/j.compositesa.2017.09.009.

URL http://www.sciencedirect.com/science/article/pii/S1359835X1730338X

[39] J. L. Park, Y.-S. Chi, T. J. Kang, Ballistic performance of hybrid panels composed of unidirectional/woven fabrics, Textile Research Journal 83 (5) (2013) 471-486. doi:10.1177/0040517512444337.

URL https://doi .org/10.1177/0040517512444337

[40] H. Shanazari, G. Liaghat, H. Hadavinia, A. Aboutorabi, Analytical investigation of high-velocity impact on hybrid unidirectional/woven composite panels, Journal of Thermoplastic Composite Materials 30 (4) (2017) 545-563. doi:10.1177/0892705715604680.

URL https://doi .org/10.1177/0892705715604680

[41] H. Liu, B. G. Falzon, W. Tan, Experimental and numerical studies on the impact response of damage-tolerant hybrid unidirectional/woven carbon-fibre reinforced composite laminates, Composites Part B: Engineering 136 (2018) 101-118. doi:10.1016/j.compositesb.2017.10.016.

URL http://www. sciencedirect.com/science/article/pii/S1359836817322059

[42] P. Navarro, J. Aubry, S. Marguet, J. F. Ferrero, S. Lemaire, 
P. Rauch, Experimental and numerical study of oblique impact on woven composite sandwich structure: Influence of the firing axis orientation, Composite Structures 94 (6) (2012) 1967-1972. doi:10.1016/j.compstruct.2012.02.001.

[43] J.-C. Passieux, P. Navarro, J.-N. Pri, S. Marguet, J.-F. Ferrero, A Digital Image Correlation Method For Tracking Planar Motions Of Rigid Spheres: Application To Medium Velocity Impacts, Experimental Mechanics 54 (8) (2014) 1453-1466. doi:10.1007/s11340-014-9930-y. URL https://link. springer.com/article/10 .1007/s11340-014-9930-y 


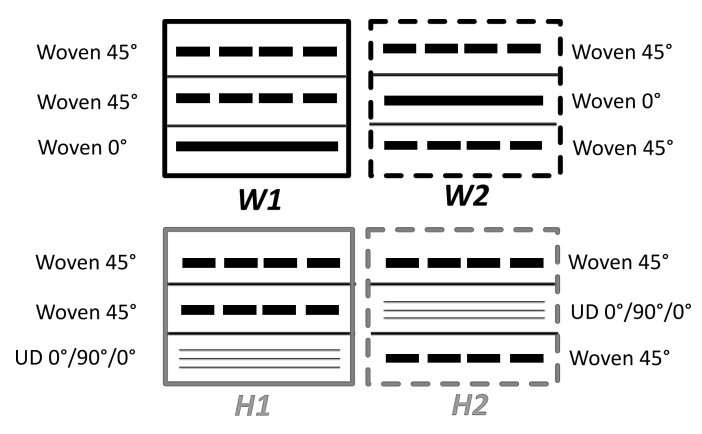

Figure 1: Stacking sequences of the four impacted configurations.

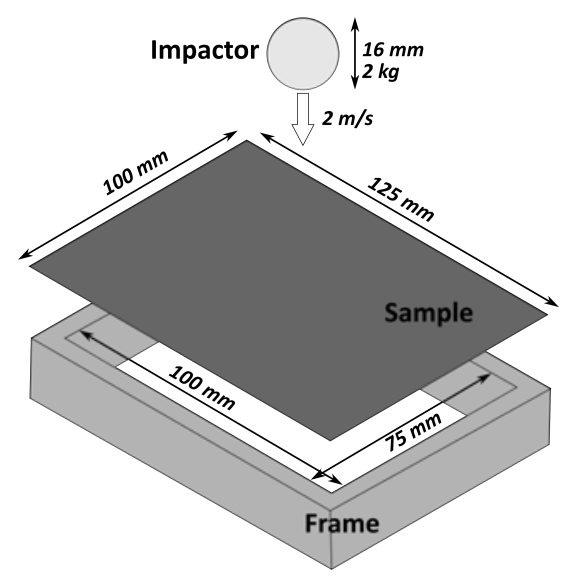

Figure 2: Boundary conditions for the low velocity impact tests. 


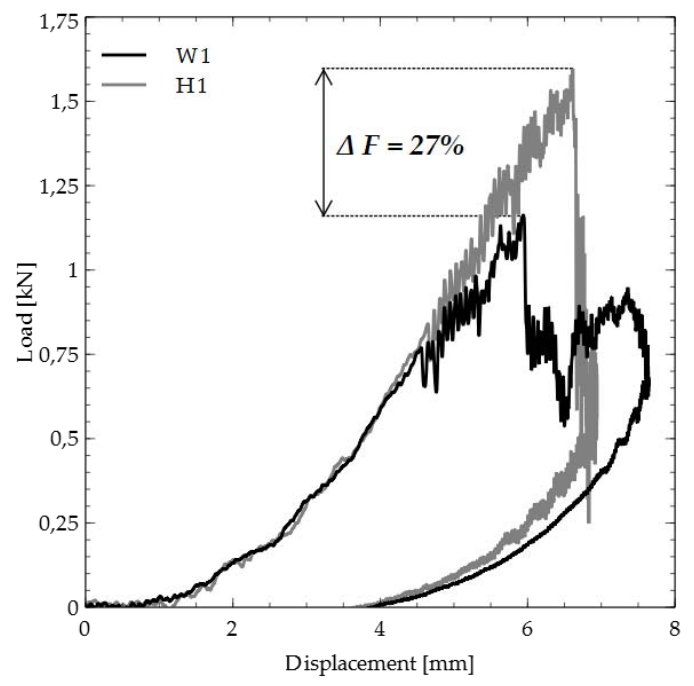

Figure 3: Load / displacement curves for H1 and W1.

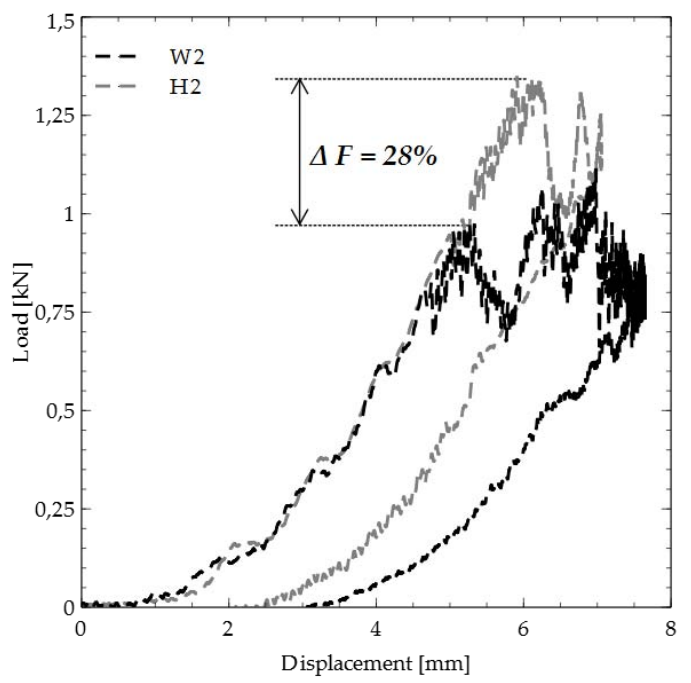

Figure 4: Load / displacement curves for H2 and W2. 


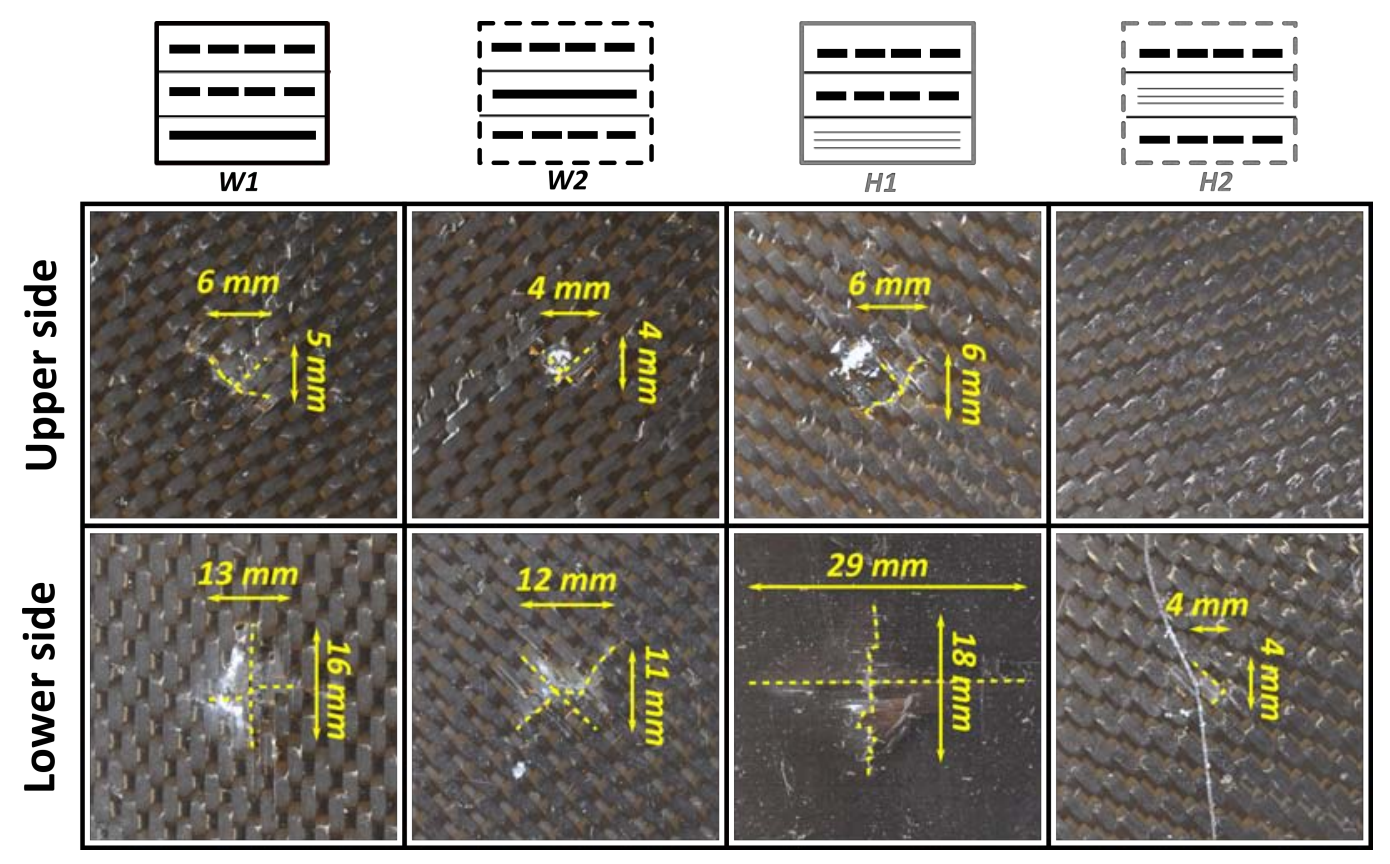

Figure 5: Low velocity post impact fracture surfaces.

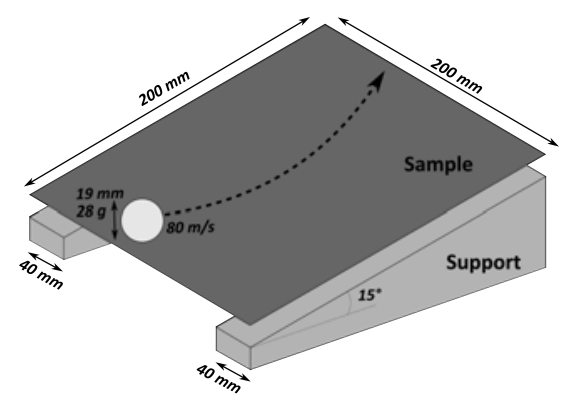

Figure 6: Boundary conditions for the medium velocity oblique impact tests. 


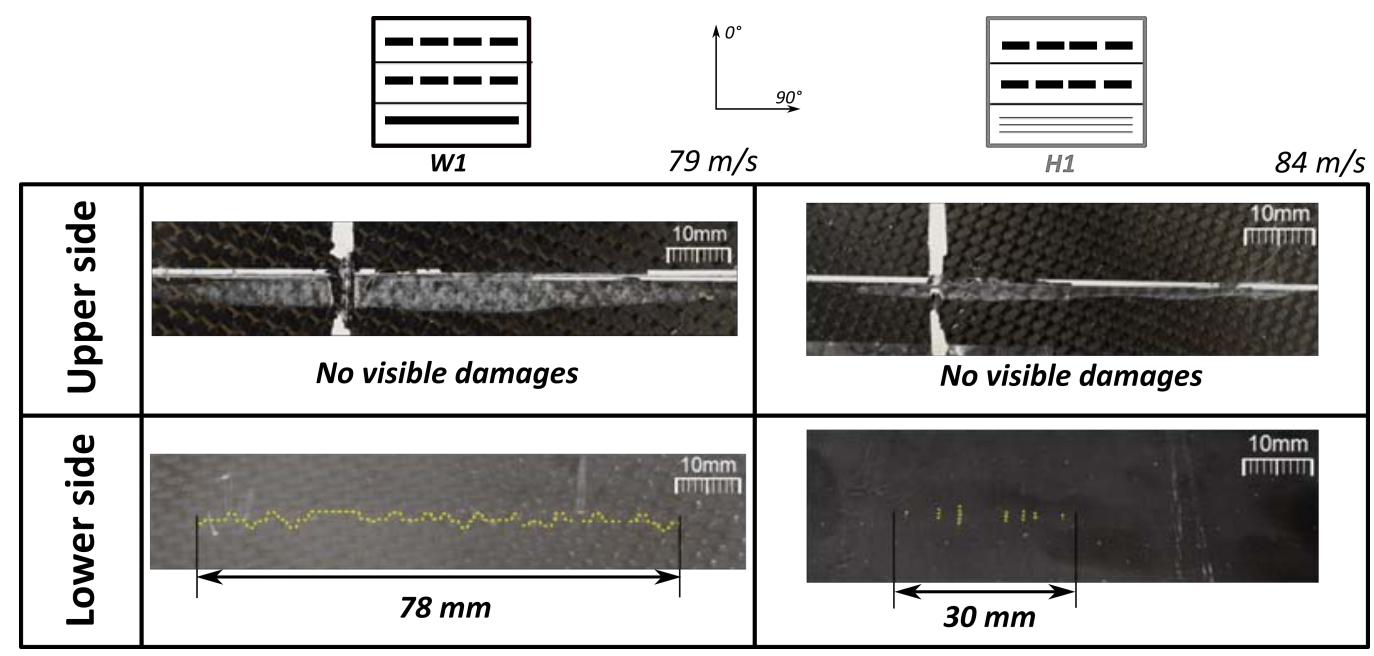

Figure 7: Medium velocity oblique impact fracture surfaces for H1 and W1.

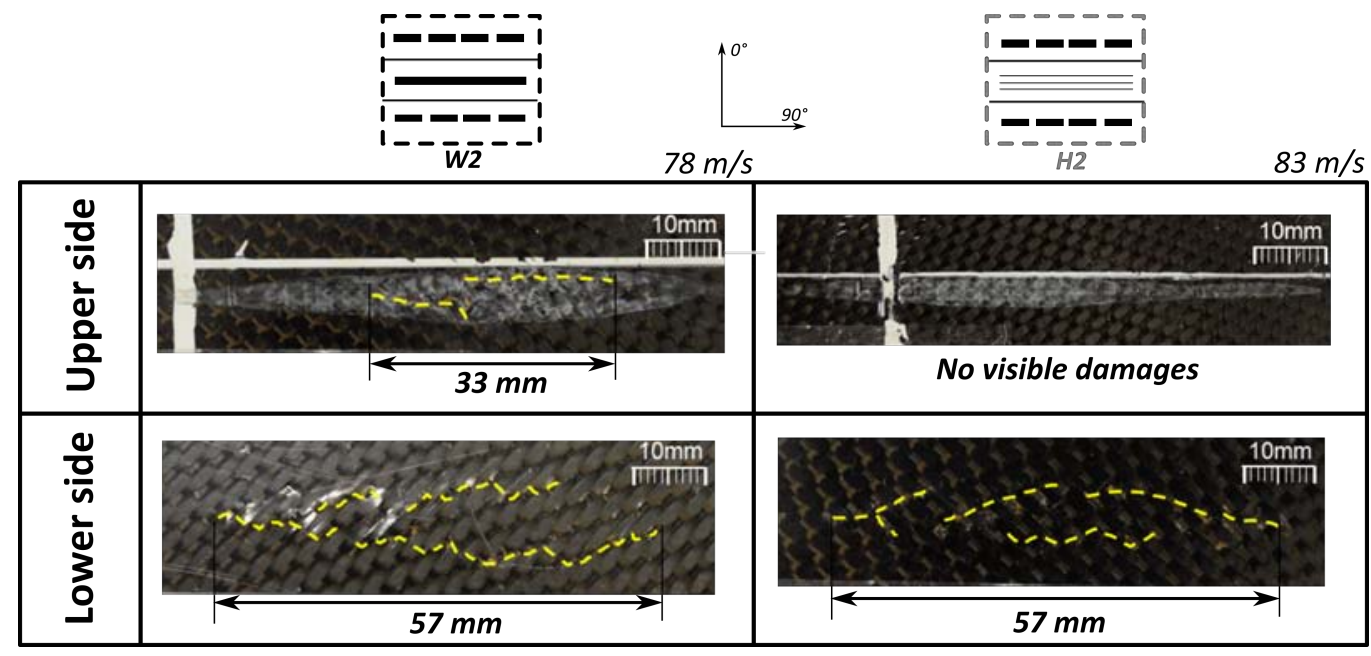

Figure 8: Medium velocity oblique impact fracture surfaces for H2 and W2. 


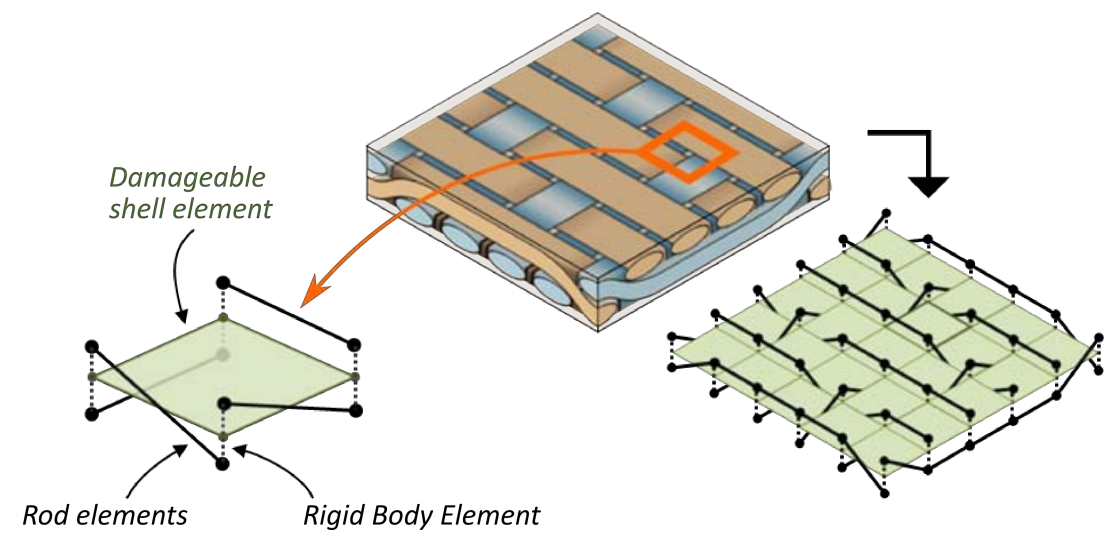

Figure 9: Principle of the Semi-Continuous modelling of woven fabrics.

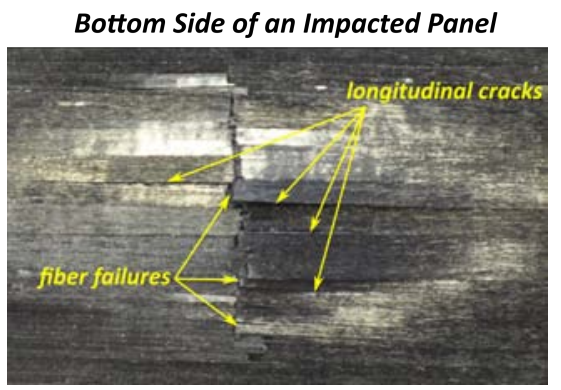

Figure 10: Example of a post-impact fracture surface of an unidirectional ply. 


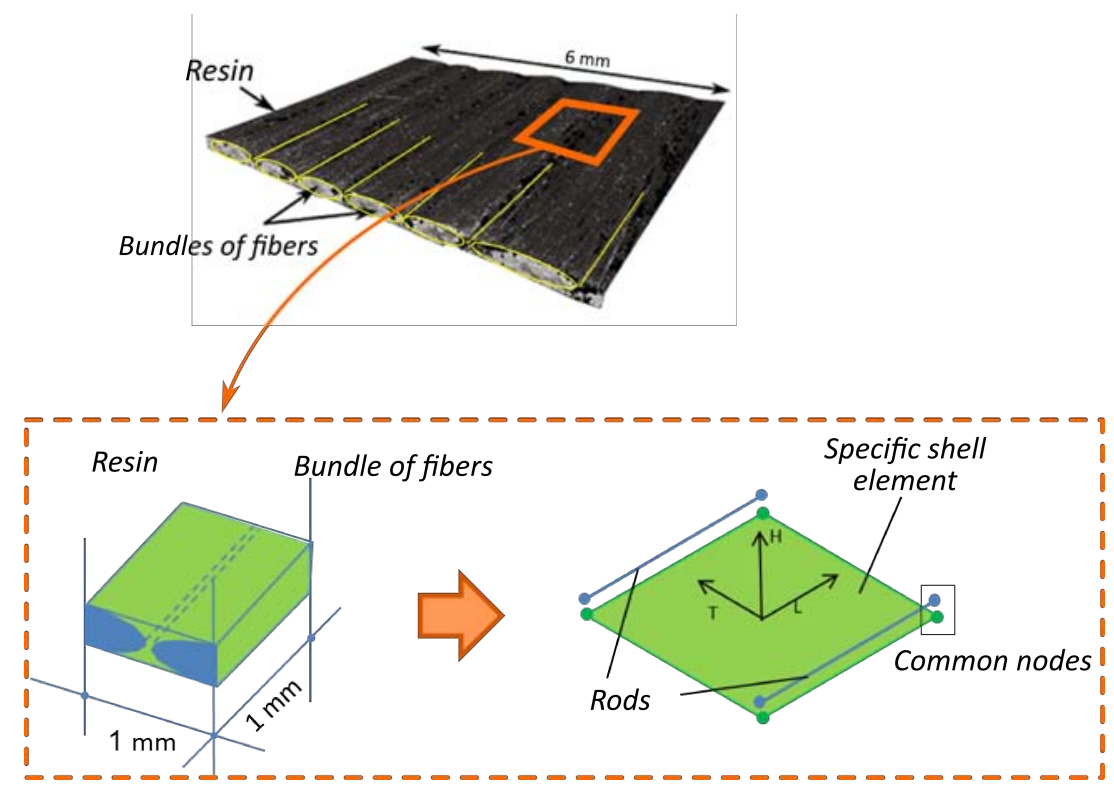

Figure 11: Principle of the Semi-Continuous modelling of an unidirectional ply.

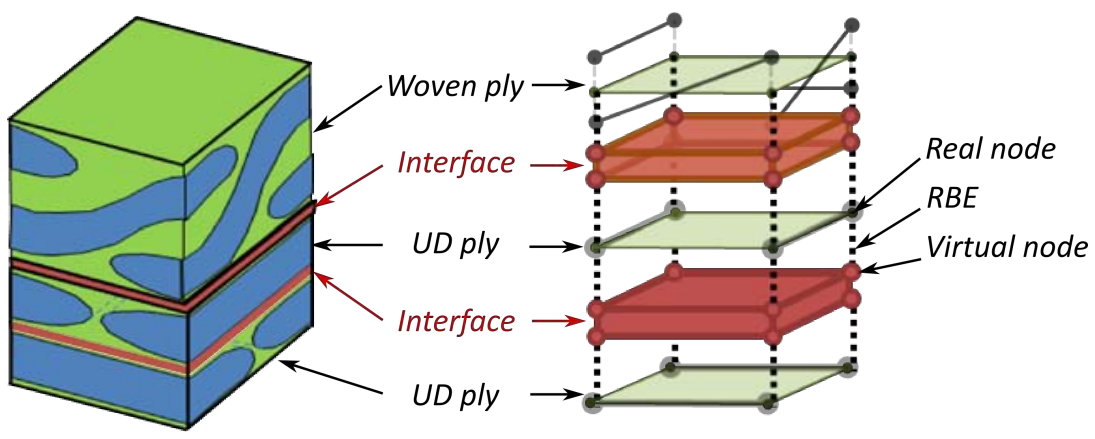

Figure 12: Principle of the Semi-Continuous modelling of the interface. 


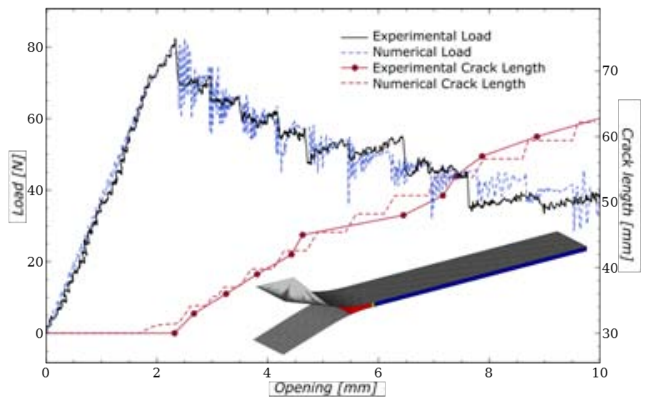

Figure 13: Example of a reverse identification for a DCB test.

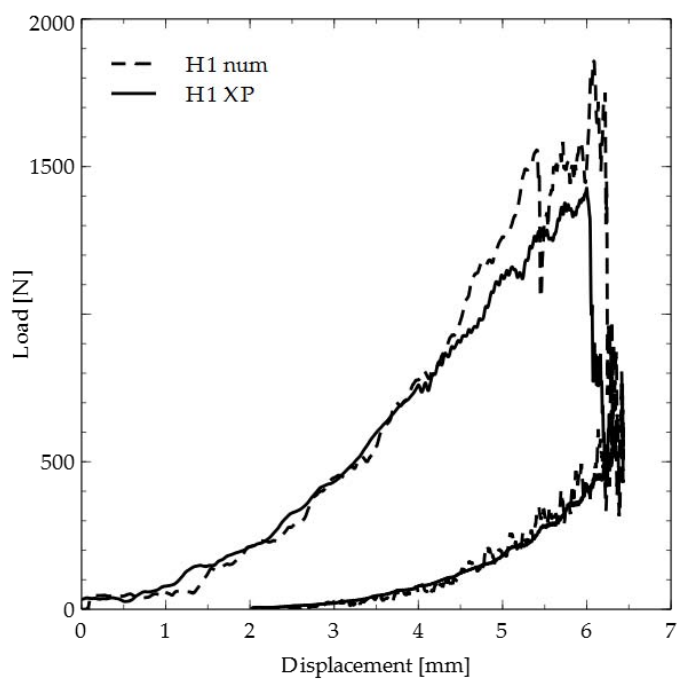

Figure 14: Experimental and numerical Load/displacement curves for a low velocity impact on the sample $\mathrm{H} 1$. 


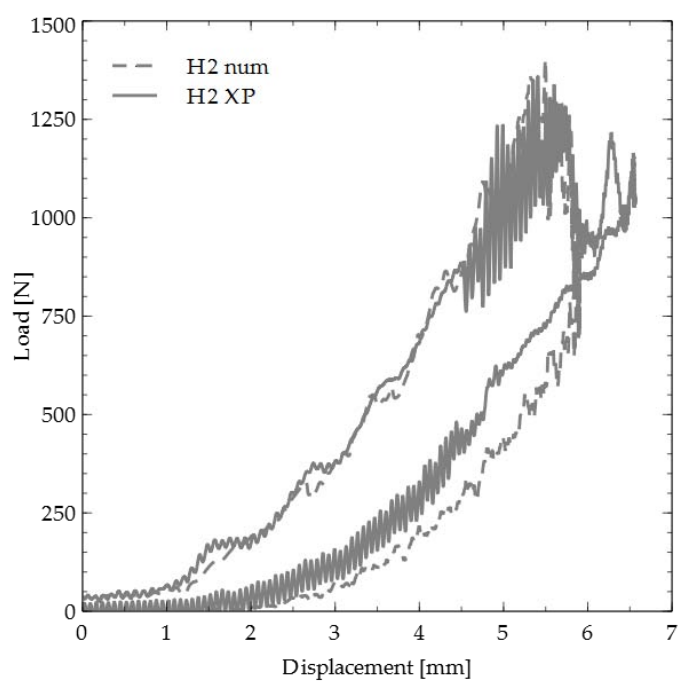

Figure 15: Experimental and numerical Load/displacement curves for a low velocity impact on the sample $\mathrm{H} 2$. 


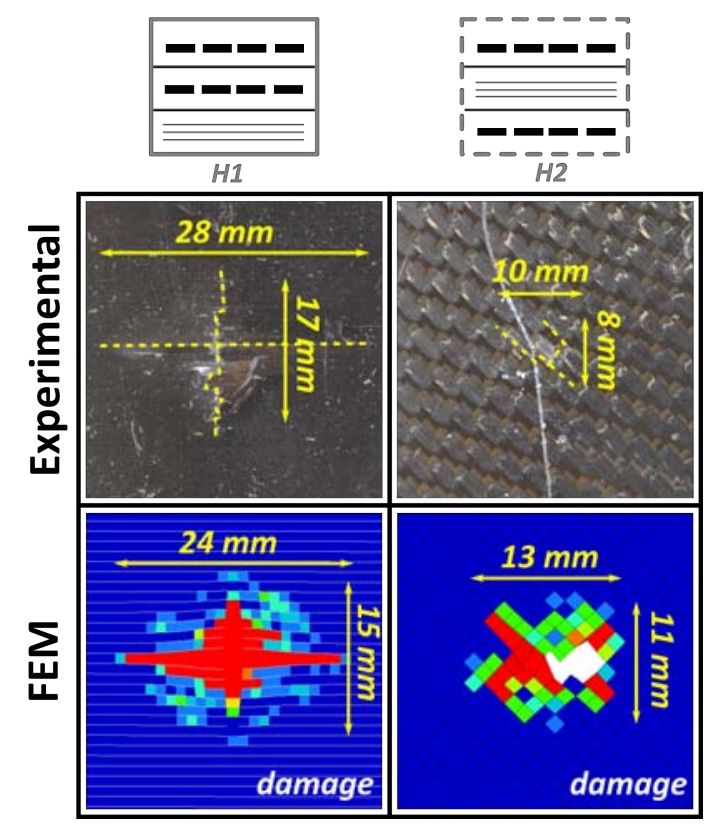

Figure 16: Experimental and numerical post impact fracture surfaces for the lower plies of hybrid samples. 


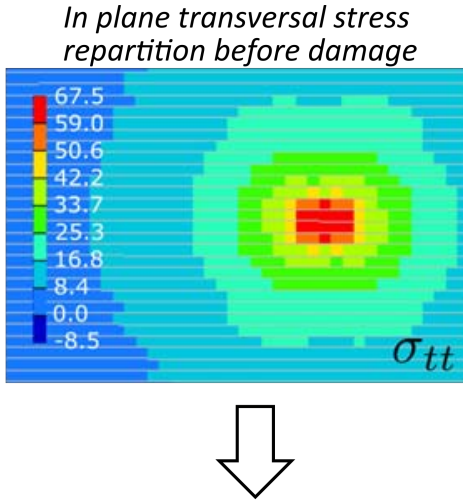

Final transversal damage and fiber ruptures

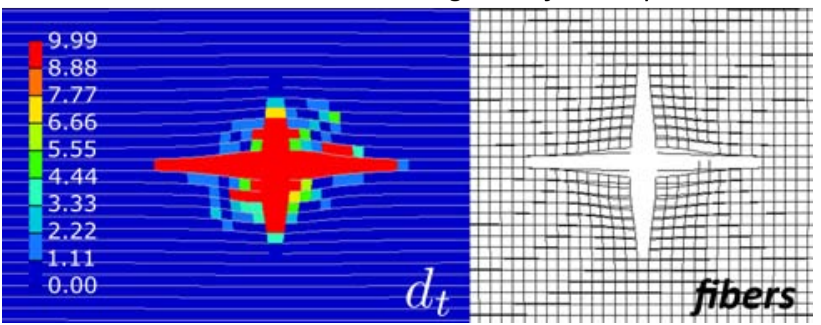

Figure 17: Mechanisms leading to the damage of the UD plies of hybrid sample H1 during a low velocity impact. 


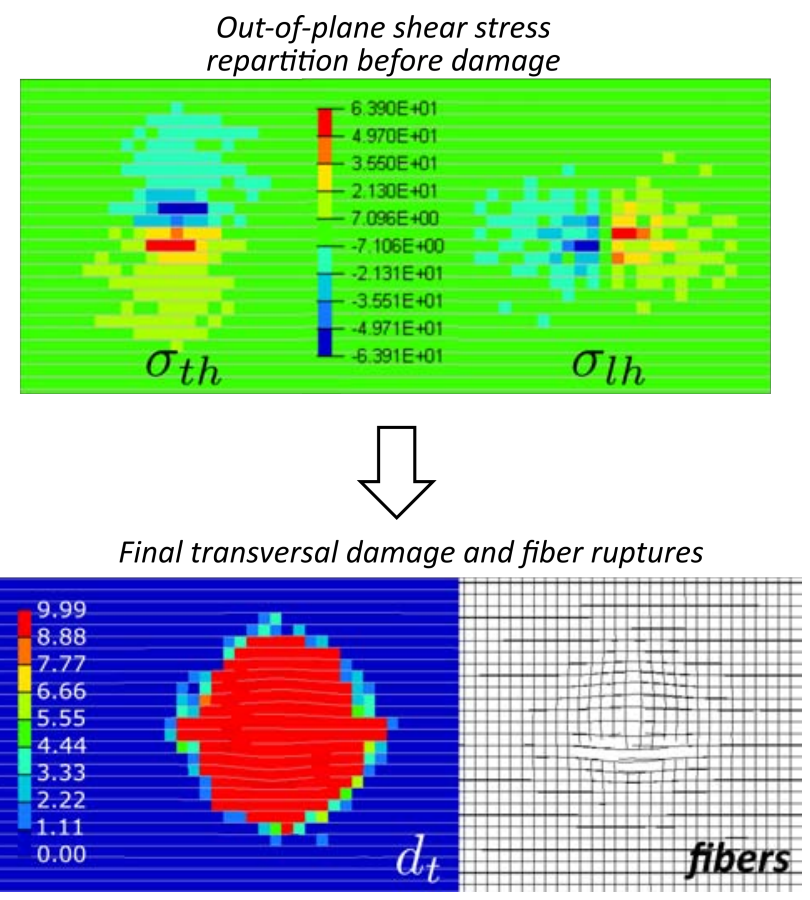

Figure 18: Mechanisms leading to the damage of the UD plies of hybrid sample H2 during a low velocity impact. 


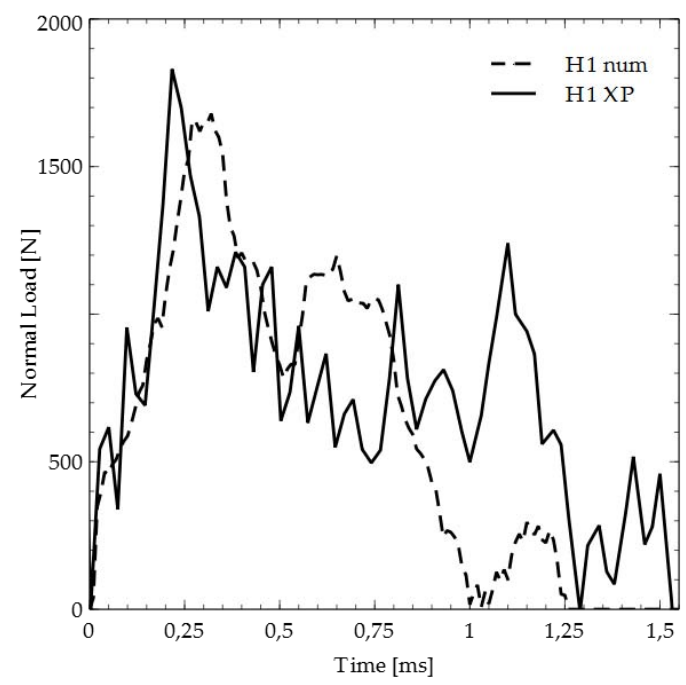

Figure 19: Experimental and numerical normal load/time curves for a low velocity impact on the sample $\mathrm{H} 1$.

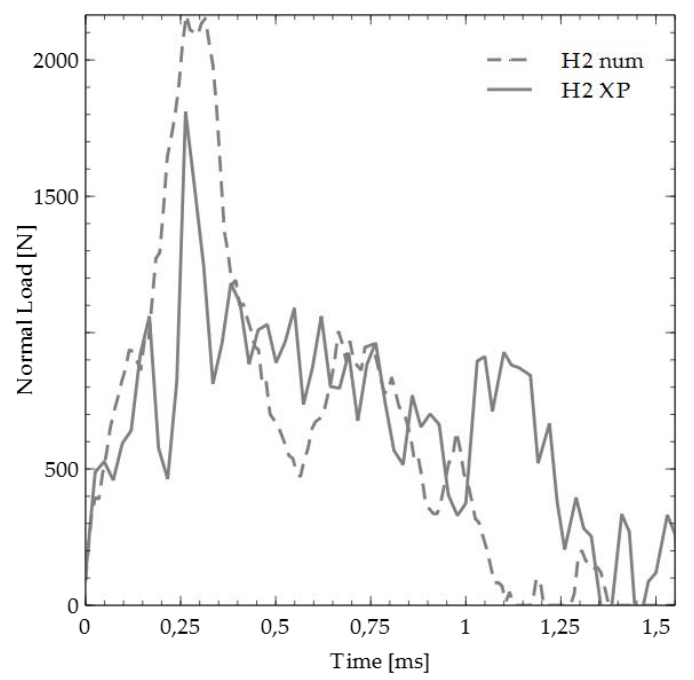

Figure 20: Experimental and numerical normal load/time curves for a low velocity impact on the sample $\mathrm{H} 2$. 


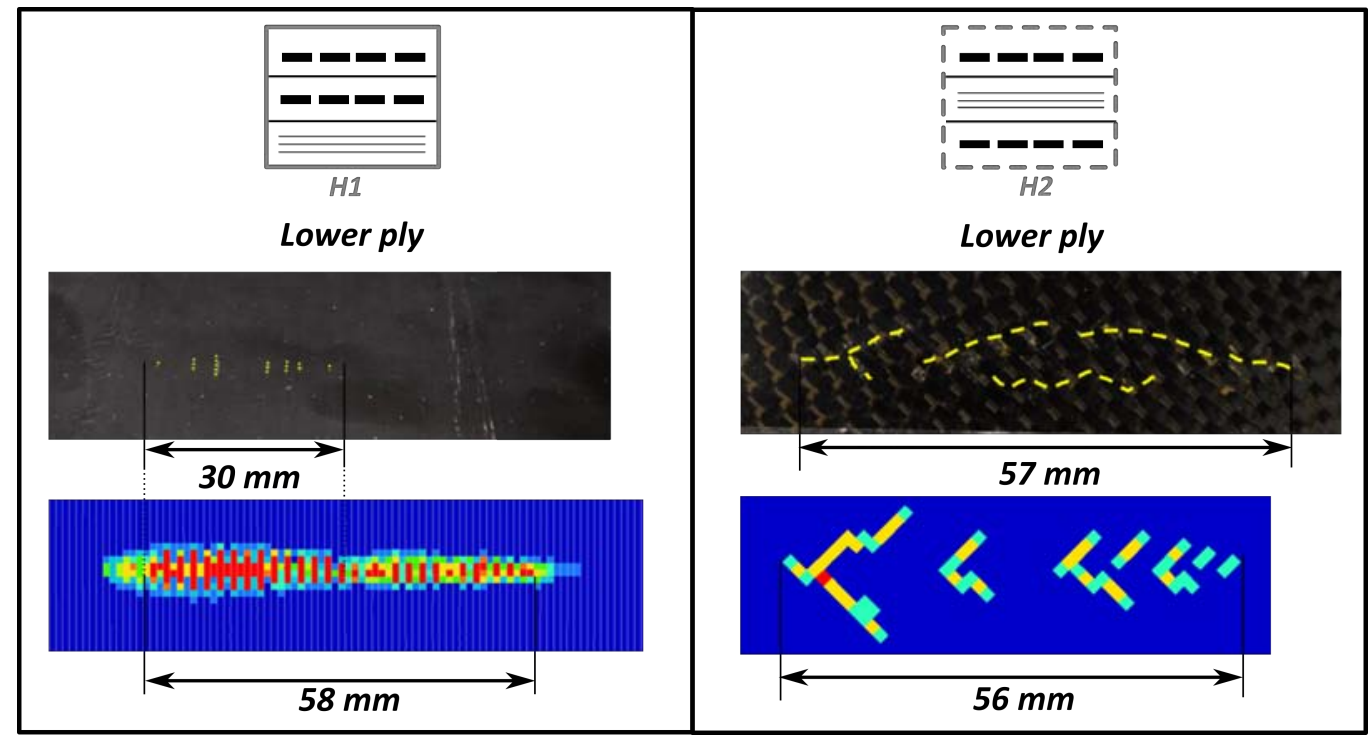

Figure 21: Experimental and numerical fracture surfaces for a medium velocity oblique impact. 

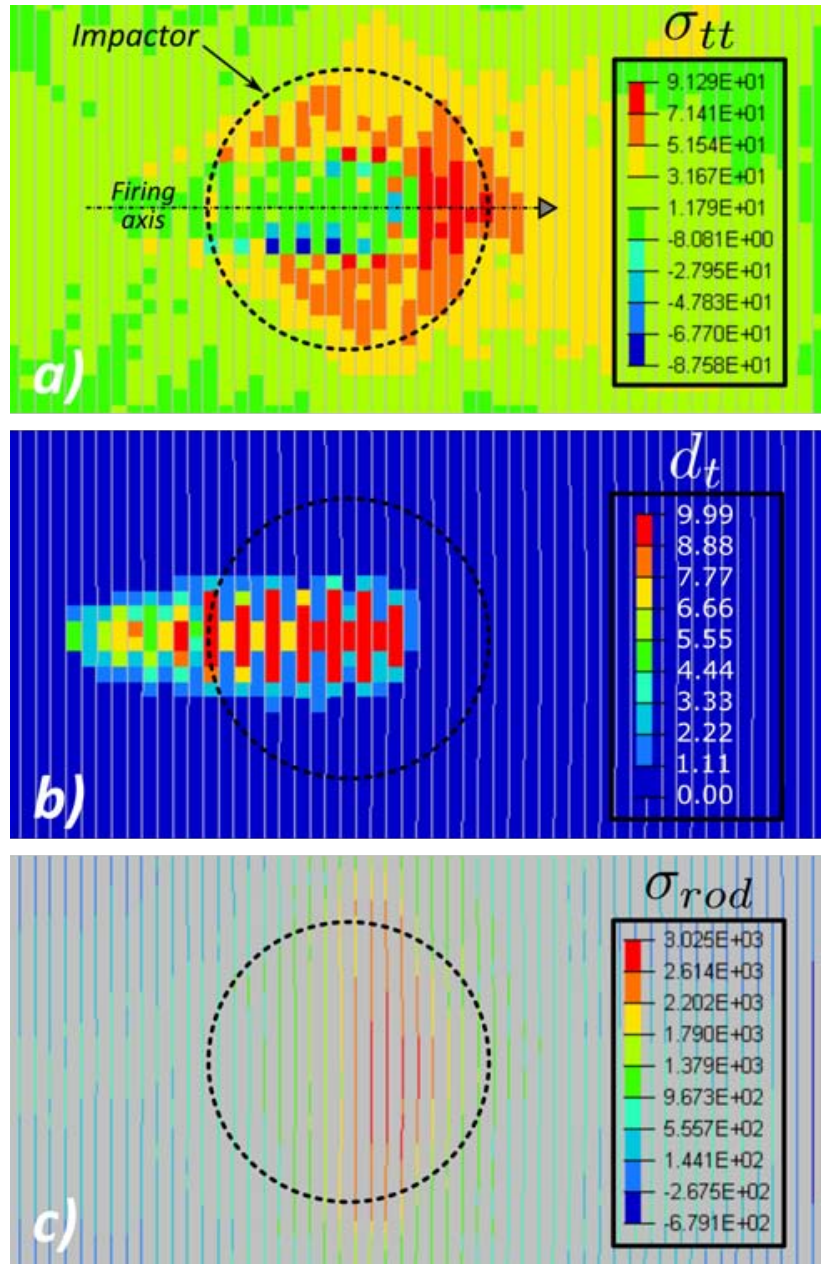

Figure 22: Mechanisms leading to the damage of the UD plies of sample H1 during a medium velocity impact. 